\title{
Beyond-mean-field correlations and the description of superheavy elements
}

\author{
Paul-Henri Heenen ${ }^{1, a}$, Benjamin Bally ${ }^{2}$, Michael Bender ${ }^{3}$, and Wouter Ryssens ${ }^{1}$ \\ ${ }^{1}$ PNTPM, CP229, Université Libre de Bruxelles, 1050 Bruxelles, Belgium \\ ${ }^{2}$ ESNT, CEA Saclay, IRFU/Service de Physique Nucléaire, 91191 Gif-sur-Yvette, France \\ ${ }^{3}$ IPNL, Université Lyon 1, CNRS/IN2P3, 69622 Villeurbanne, France
}

\begin{abstract}
The role of beyond-mean-field correlations from the restoration of broken symmetries and configuration mixing for the description of superheavy elements is illustrated.
\end{abstract}

\section{Introduction}

Self-Consistent Mean-Field (SCMF) models are one of the standard approaches to describe a wide range of phenomena in nuclear structure and dynamics [1]. They are the only available microscopic method that at present can be applied in a systematic manner to all bound atomic nuclei irrespective of their mass and their proton-to-neutron ratio. For superheavy elements (SHE) this method is even the only microscopic model presently available for large-scale studies [2-4].

In one way or the other, the product state that provides the solution of the SCMF equations always breaks some symmetries of the nuclear Hamiltonian, for example through deformation of the density distribution or the presence of BCS-type pairing correlations. This feature is at the heart of the predictive and explanatory power of the nuclear SCMF method, as it can be exploited to grasp correlations associated with collective degrees of freedom in an intuitive manner at moderate computational cost. The physical many-body states, however, are eigenstates of angular momentum, parity, proton and neutron numbers, which in general might all be broken by SCMF states. For some applications, however, it is important to restore the states' quantum numbers within what is usually called a "beyondmean-field method". Exact projection on angular momentum, parity, and particle number then ensures that calculated energy spectra and transition moments for electromagnetic and other transitions can be unambiguously compared with experimental data. Similarly, for many nuclei the mixing of states with different intrinsic deformation is necessary for their correct description, for example to describe fluctuations in shape degrees of freedom or the widespread phenomenon of shape coexistence. Such mixing can be most easily achieved within the Generator Coordinate Method (GCM), which can be naturally combined with projection into a single framework $[5,6]$.

The beyond-mean-field method that we use has already been applied to many regions of the nuclear chart [7-10]. A complete calculation proceeds in three steps. The first one consists in carrying out a set of mean-field calculations with suitably varied constraints on the shape of the nucleus. The resulting intrinsic wave functions are then projected on particle numbers and

\footnotetext{
${ }^{a}$ Corresponding author: phheenen@ulb.ac.be
}

(C) The Authors, published by EDP Sciences. This is an Open Access article distributed under the terms of the Creative Commons Attribution License 4.0 (http://creativecommons.org/licenses/by/4.0/). 


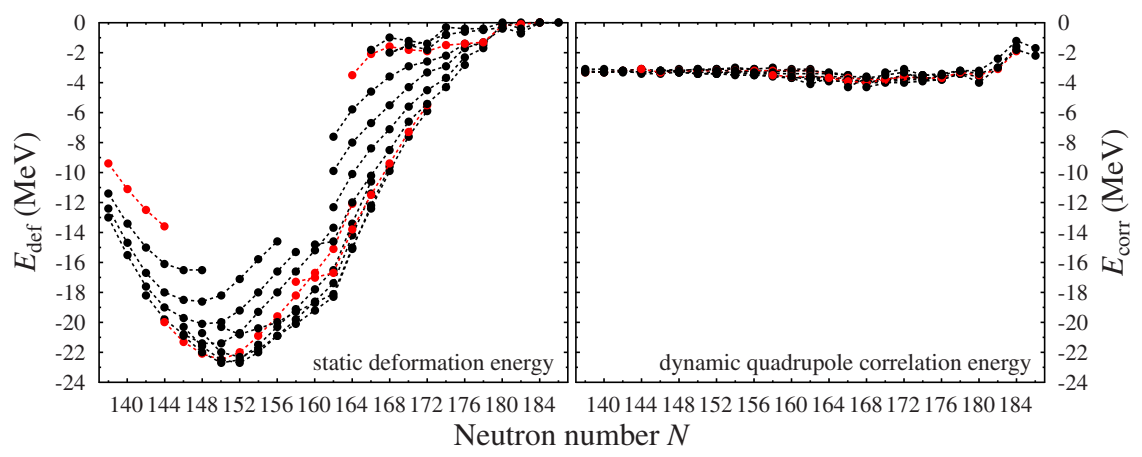

Figure 1. Static and dynamic deformation energies (see text) of heavy and superheavy nuclei around the valley of stability, plotted as a function of their neutron number. Lines connect nuclides within an isotopic chain. Those marked in red correspond to $Z=90,100,110$, and 120, see Fig. 2.

angular momentum. Finally, the symmetry restored states are mixed. The final result of such a calculation is a spectrum of states in the laboratory frame of reference. In what follows, we will give a few illustrative results for properties of very heavy and superheavy nuclei obtained at the various stages of such method.

\section{Systematic study of even-even SHE}

A systematic beyond-mean-field calculation of even-even superheavy nuclei has been carried out under the same conditions as in the earlier study [7] that addressed the properties of lighter even-even nuclei up to $A \approx 250$. The SLy4 parameterisation of the Skyrme EDF is used throughout. The mean-field configurations are limited to axial shapes with conserved parity, which, after projection on good particle number and angular momentum $J=0$, are then mixed within the GCM.

The resulting static and the dynamic deformation energies are plotted in Fig. 1. The static deformation energy is defined as the difference between the binding energy obtained for the spherical mean-field configuration and the lowest energy found when allowing for deformed shapes. The dynamic deformation energy is the additional gain obtained for the $J^{\pi}=0^{+}$ground state after projection and configuration mixing, see also Ref. [7]. The static deformation energy can reach up to $22.7 \mathrm{MeV}$ and varies quickly from one isotope to the other. By contrast, the variation of the dynamic correlation energy is less structured. As in earlier studies [4, 11-13], spherical mean-field ground states are only found in the direct vicinity of the neutron shell closure at $N=184$, while nuclei with a proton shell closure, which for SLy4 is at $Z=126$, are found to be deformed, with deformation energies of a few MeV. As observed for lighter nuclei in Ref. [7], the dynamic correlation energy plays a crucial role only for transitional nuclei close to spherical shell closures, where it is of the same order or even larger than the static deformation energy. In such case, it might change the topography of energy surfaces and visibly modify binding energy differences [7].

For superheavy nuclei, the most important binding energy difference is the $Q_{\alpha}$ value, which often is the first observable that becomes available for newly discovered nuclides. Figure 2 presents results for $Q_{\alpha}$ values obtained at four different levels of modelling. The upper left panel shows $Q_{\alpha}$ values obtained from a liquid drop model (LDM) whose parameters correspond to the nuclear matter properties of the EDF used for the microscopic calculations [14]. No structure can be seen; the very smooth global trends are entirely determined by the scaling of Coulomb and symmetry energy with proton and neutron number. 


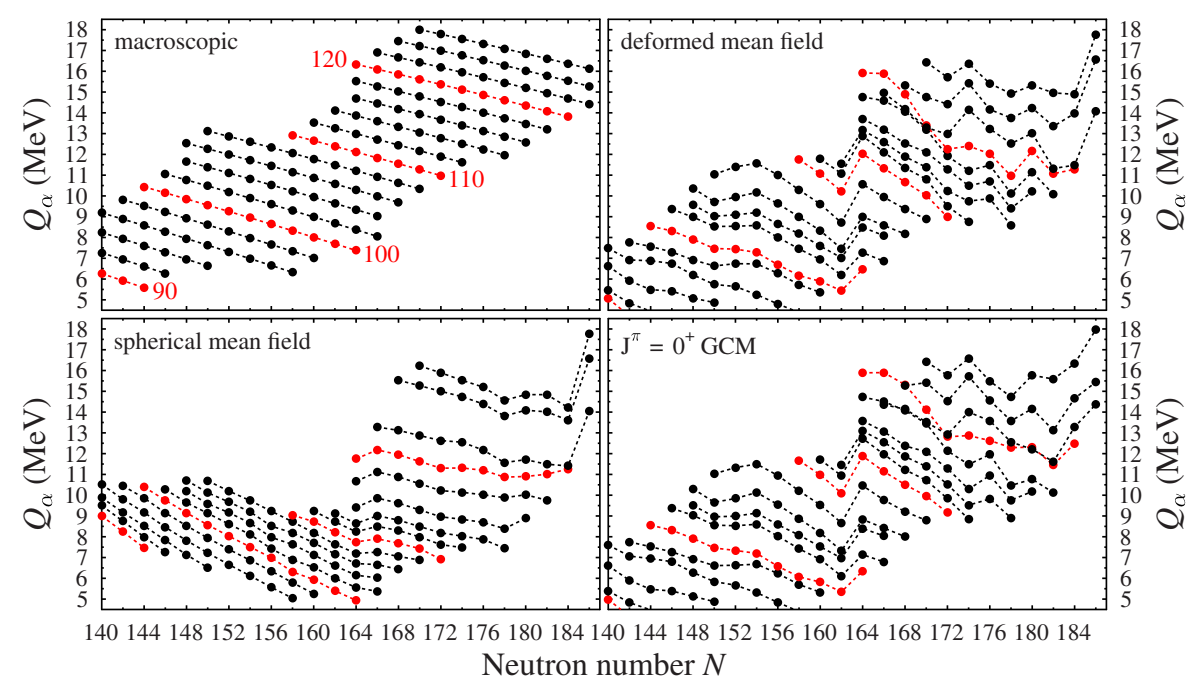

Figure 2. $Q_{\alpha}$ values obtained at four different levels of modelling (see text). Lines connect nuclides within isotopic chains. Those drawn in red correspond to $Z=90,100,110$, and 120 as indicated in the upper left panel.

The effect of spherical shell structure can be seen by comparing with the lower left panel that displays the $Q_{\alpha}$ values obtained when imposing spherical symmetry on the mean-field states. When plotted as in Fig. 2, large jumps within an isotopic chain indicate a neutron shell effect, whereas large gaps between the isotopic chains indicate a proton shell effect. Not all of those visible are related to actual shell closures, however. As pointed out in Ref. [15], spherical configurations of superheavy nuclei have the unusual feature that variations in the degeneracy of spherical shells around the Fermi energy, which result from the sequence of high- $j$ and low- $j$ orbits in the spectrum, are sufficient to provide significant extra binding without the appearance of visible shell closures. And indeed, the spreading of the curves representing spherical $Q_{\alpha}$ values for $Z>114$ cannot be correlated to the opening of gaps in the single-particle spectrum, see Fig. 3. Instead, it results from the small degeneracy of the low- $j$ orbits around the Fermi energy. Comparing this panel with the upper right one shows the effect of static quadrupole deformation. As most nuclei are deformed, the density of single-particle levels is much more uniform than for spherical shapes. As a result, the $Q_{\alpha}$ values from deformed mean-field calculations are on the average much closer to the LDM values than to those from spherical mean-field calculations. In the region of nuclei with large deformation energy, the remaining jumps and gaps between the lines indicate deformed neutron $(N=150,162)$ and proton $(Z=108)$ shell closures, not all of which agree with experiment $[3,12,16]$. For the heavier transitional nuclei the direct connection between shell structure and $Q_{\alpha}$ remains less direct. As expected from Fig. 1, the dynamic quadrupole correlations obtained from constructing the $J=0$ projected GCM ground states only have a minor impact on $Q_{\alpha}$ values, see the lower right panel. Still, compared to the deformed meanfield results, for the heavy transitional nuclei all curves are slightly shifted in a non-uniform manner, most obviously for the $Z=120$ chain drawn in red. How these calculated values compare with experiment has already been discussed in Ref. [4].

The interplay of single-particle shell structure, ground-state deformation and dynamic quadrupole collectivity in these calculations is illustrated in Fig. 3 for ${ }^{290} 116$ and ${ }^{294} 118$, the two heaviest even-even nuclei identified so far [17]. When calculated with the SLy4 


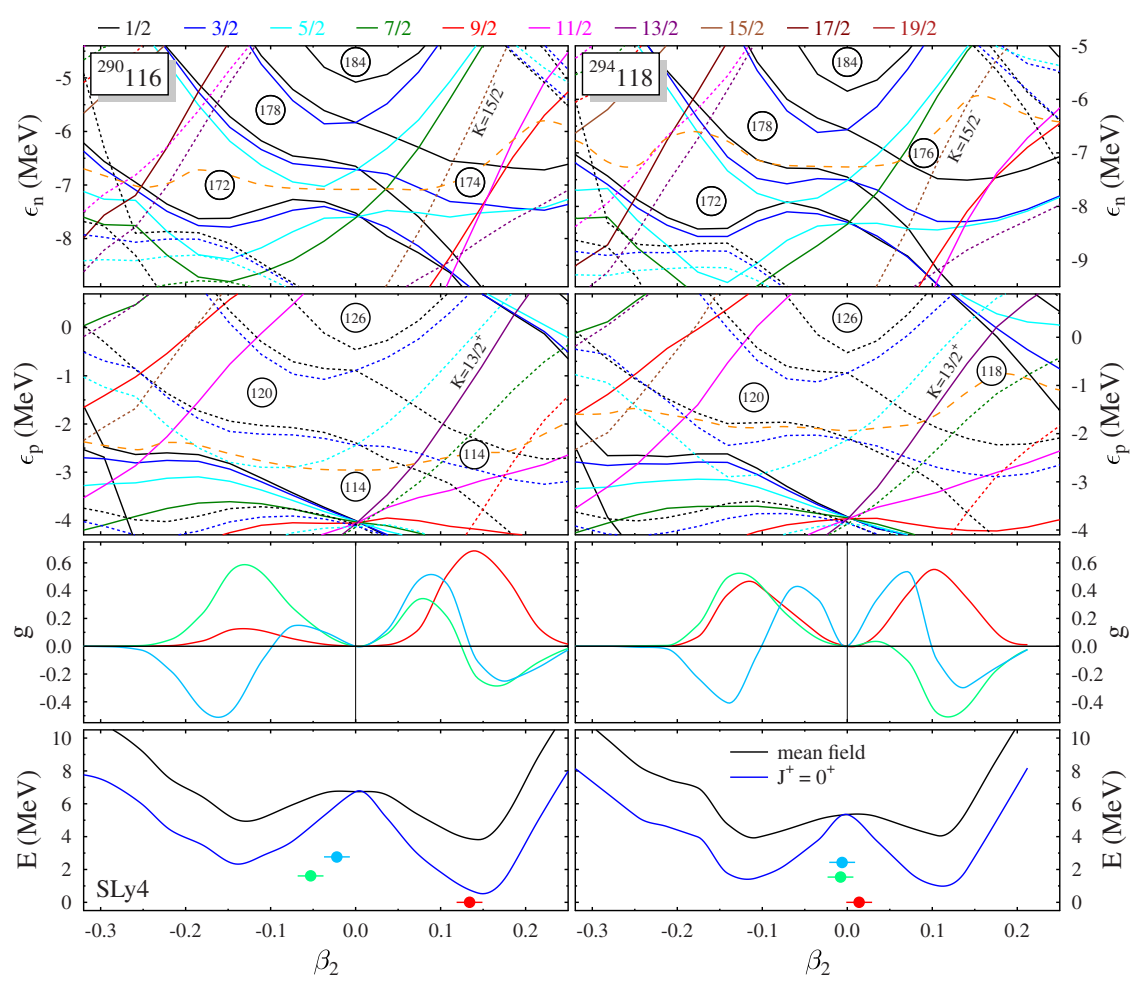

Figure 3. From top to bottom: Nilsson diagrams of neutron $\left(\epsilon_{n}\right)$ and proton $\left(\epsilon_{p}\right)$ single-particle energies, collective GCM wave functions $g$ of the three lowest $0^{+}$states, and the mean field (black) and angular momentum $J=0$ projected deformation energy curves obtained for ${ }^{290} 116$ (left panels) and ${ }^{294} 118$ (right panels) as a function of the intrinsic axial quadrupole deformation $\beta_{2}$. For the definition of $\beta_{2}$ and $g\left(\beta_{2}\right)$ see Ref. [7]. Positive (negative) $\beta_{2}$ values indicate prolate (oblate) shapes. The colour of the levels in the Nilsson diagrams indicates the expectation value of angular-momentum along the nucleus' symmetry axis. Solid (dotted) lines correspond to levels of positive (negative) parity. The respective Fermi energies are indicated by dashed orange lines. The numbers put into various gaps in the Nilsson diagrams indicate the number of particles that can be put into the levels below. The bottom panel also show the energies of the GCM states drawn at the average deformation of the intrinsic states they are constructed from using the expressions from Ref. [7]. The corresponding wave functions $g\left(\beta_{2}\right)$ in the panel above are plotted in the same colour.

parameterisation of the Skyrme EDF, both nuclei have soft deformation energy surfaces with slightly deformed minima. Calculations of the full $\beta-\gamma$ plane that will be reported elsewhere [19] indicate that the prolate and oblate minima visible for ${ }^{294} 118$ remain separated by a small barrier in $\gamma$ direction, while for ${ }^{290} 116$ the oblate structure is actually a saddle point. The energy gain from projection on angular momentum $J=0$ increases with deformation, which for ${ }^{294} 118$ inverts the order of the two minima.

The collective GCM wave functions of the lowest $0^{+}$states are significantly different in both nuclei and illustrate the distinction between deformation softness $\left({ }^{294} 118\right)$ and shape coexistence $\left({ }^{290} 116\right)$. The wave functions of ${ }^{294} 118$ are almost equally spread over both minima. By contrast, the ground state of ${ }^{290} 116$ is mainly situated on the prolate side, whereas its first excited $0^{+}$is mainly oblate. The origin of this difference can be identified in the Nilsson diagram. As has been argued for a long time, the phenomenon of shape coexistence relates to configurations with a different number of occupied intruder orbitals [18], i.e. singleparticle states with a parity opposite to the one of the last major shell. The Nilsson diagrams 

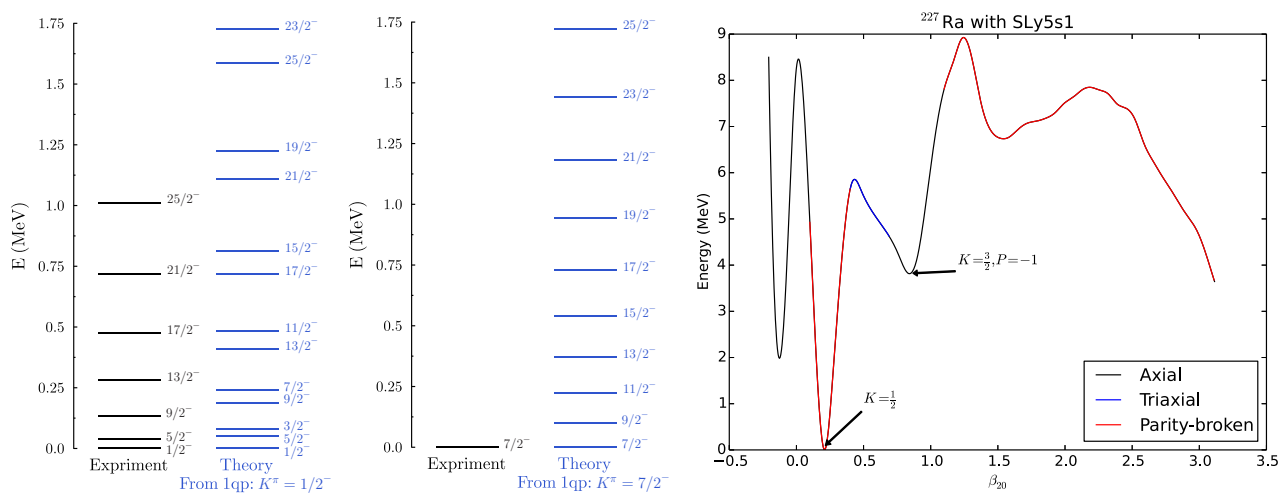

Figure 4. Left: energies obtained after projection on angular momentum and particle numbers of two different one-quasiparticle states of ${ }^{251} \mathrm{Md}$. Right: fission barrier for ${ }^{227} \mathrm{Ra}$ constructed from the lowest one-quasiparticle configuration found at each deformation. Colours indicate the symmetries taken by the solutions found.

of both nuclei are very similar, although there are many small differences in the relative position of the single-particle levels. The main difference relevant for our discussion is the change in position of the Fermi energies that indicate where the smooth distributions of occupation numbers as determined by pairing correlations fall below $1 / 2$. Its shift has two important consequences for configurations around the prolate minimum. Firstly, the up-sloping neutron $K^{\pi}=15 / 2^{-}$level originating from the spherical $1 j_{15 / 2^{-}}$intruder subshell is still below the Fermi energy for ${ }^{294} 118$, but far above for ${ }^{290} 116$. Secondly, the $K^{\pi}=13 / 2^{+}$proton level from the spherical $i_{13 / 2^{+}}$intruder sub-shell is next to the Fermi energy for ${ }^{294} 118$, but much higher up for ${ }^{290} 116$. There are no other intruder levels crossing the Fermi energy in the range of deformations over which the lowest $0^{+}$states are spread. As a consequence, about the same number of proton and neutron intruder levels has large occupancy on the prolate and the oblate sides for ${ }^{294} 118$, whereas for prolate configurations of ${ }^{290} 116$ there are roughly two neutron and proton intruder orbitals less that are occupied for oblate ones, which leads to two distinct configurations that are only weakly mixed in ${ }^{290} 116$. Such difference might have a visible impact on the $\alpha$ transition probability; however, no significant deviation from the empirical relation between $Q_{\alpha}$ values and lifetimes is found for the observed transition between both nuclei [17]. A more detailed analysis of these results will be given elsewhere [19].

\section{Prospective calculations for odd-mass nuclei}

The application of beyond-mean-field methods to odd nuclei is still in its infancy and requires new developments in particular to build effective interactions. The need to block one-quasiparticle states makes their description already much more complicated at the level of SCMF calculations.

As a first application of the projection techniques for odd nuclei that we have recently developed [10] to very heavy nuclei, the left panel of Fig. 4 shows the spectra obtained from the projection of two different blocked one-quasiparticle HFB configurations obtained for ${ }^{251} \mathrm{Md}$ with the effective interaction SLyMR0 [20]. There is a clear difference between the spectra obtained from the projection of these two near-axial states with $K^{\pi} \approx 7 / 2^{-}$and $K^{\pi} \approx 1 / 2^{-}$, respectively. While the former is very regular, a very large signature splitting is found for the latter. Only levels in the favoured band built on the $K^{\pi} \approx 1 / 2^{-}$level have 
been observed so far [21]; compared to the data, the projected energy spectrum is sligthly too spread out.

Besides $\alpha$ decay, fission is the overall most important decay mode of superheavy nuclei. While there are many calculations of the fission barriers of heavy even-even nuclei, there are almost none for odd ones. How to follow their fission path is not a trivial problem. Indeed, the one-quasiparticle configuration that provides the lowest energy may change its properties along the fission path, making it much more complicated to ensure that the path is continuous. Non-axial deformations can connect axial configurations with different $K$-value, while octupole deformations mix states of opposite parity. The fission barrier of ${ }^{227} \mathrm{Ra}$ as obtained from exploratory blocked HFB calculations [22] with the recent SLy5s1 parameterization of the Skyrme EDF [23] is plotted in the right panel of Fig. 4. At each deformation the energy of the lowest configuration found is given. For the ground state of this nucleus, parity is broken and the lowest quasiparticle corresponds to $K=1 / 2$, while at large deformations, the second minimum corresponds to $K^{\pi}=3 / 2^{-}$.

Part of the computations were performed using HPC resources from of the MCIA (Mésocentre de Calcul Intensif Aquitain) of the Université de Bordeaux and of the Université de Pau et des Pays de l'Adour. This research has been supported in part by the PAI-P7-12 of the Belgian Office for Scientific Policy.

\section{References}

[1] M. Bender, P.-H. Heenen, P.-G. Reinhard, Rev. Mod. Phys. 75, 121 (2003).

[2] M. Bender and P.-H. Heenen, J. Phys. Conf. Series 420, 012002 (2013).

[3] J. Dobaczewski, A.V. Afanasjev, M. Bender, L.M. Robledo, Y. Shi, Nucl. Phys. A944, 388 (2015).

[4] P.-H. Heenen, J. Skalski, A. Staszczak, D. Vretenar, Nucl. Phys. A944, 415 (2015).

[5] M. Bender, Eur. Phys. J. ST 156, 217 (2008).

[6] J. L. Egido, Phys. Scripta 91, 073003 (2016).

[7] M. Bender, G.F. Bertsch, P.-H. Heenen, Phys. Rev. C 73, 034322 (2006).

[8] M. Bender and P.-H. Heenen, Phys. Rev. C 78, 024309 (2008).

[9] J.M. Yao, M. Bender, P.-H. Heenen, Phys. Rev. C 87, 034322 (2013).

[10] B. Bally, B. Avez, M. Bender, P.-H. Heenen, Phys. Rev. Lett. 113, 162501 (2014).

[11] S. Ćwiok, J. Dobaczewski, P.-H. Heenen, P. Magierski, W. Nazarewicz, Nucl. Phys. A611, 211 (1996).

[12] T. Bürvenich et al., Eur. Phys. J. A3, 139 (1998).

[13] S. Ćwiok, P.-H. Heenen, W. Nazarewicz, Nature, 433, 705 (2005).

[14] P.-G. Reinhard, M. Bender, W. Nazarewicz, T. Vertse, Phys. Rev. C 73, 014309 (2006).

[15] M. Bender, W. Nazarewicz, P.-G. Reinhard, Phys. Lett. B515, 42 (2001).

[16] M. Asai, F.P. Heßberger, A. Lopez-Martens, Nucl. Phys. A944, 308 (2015).

[17] Yu.Ts. Oganessian and V.K. Utyonkov, Nucl. Phys. A944, 62 (2015).

[18] K. Heyde and J.L. Wood, Rev. Mod. Phys. 83, 1467 (2011).

[19] M. Bender and P.-H. Heenen, in preparation.

[20] J. Sadoudi, M. Bender, K. Bennaceur, D. Davesne, R. Jodon, T. Duguet, Phys. Scripta T154, 014013 (2013).

[21] A. Chatillon et al., Phys. Rev. Lett. 98, 132503 (2007).

[22] W. Ryssens, Thesis, Université Libre de Bruxelles (2016).

[23] R. Jodon, M. Bender, K. Bennaceur, J. Meyer, preprint arXiv: 1606. 01410. 